\title{
The effects of travel information presentation through nomadic systems on driver behaviour
}

\author{
K. A. Brookhuis • M. Dicke
}

Received: 25 August 2008 / Accepted: 3 April 2009/Published online: 8 May 2009

(C) European Conference of Transport Research Institutes (ECTRI) 2009

\begin{abstract}
Introduction The aim of this study was to investigate the effects of providing travel information to drivers about a traffic jam ahead and a potential detour or short-cut. Two groups of participants, native and non-native Dutch speakers were requested to drive in a driving simulator under both calm and dense traffic conditions.

Method Travel-information was presented by means of three nomadic systems; in visual mode on a PDA and on a mobile phone via SMS, and through a mobile phone in auditory mode via the (simulator mock-up) vehicle's audio system.

Results The results showed that with regard to usability the SMS message was evaluated worse than the other two systems, while with respect to cognitive processing, SMS caused more subjective (i.e. experienced) workload than the other two systems. Native participants believed any information-providing system to be less useful than nonnative participants did. All participants remembered more of the information when traffic was dense whereas natives remembered more than non-natives. With regard to performance and safety, driving performance was better when traffic was calm, as compared to dense traffic; however, compensation was shown by lowering driving speed in the
\end{abstract}

\section{K. A. Brookhuis}

Experimental \& Work Psychology, University of Groningen, Grote Kruisstraat 2/1,

9712 TS Groningen, The Netherlands

K. A. Brookhuis $(\square)$

Delft University of Technology,

Delft, The Netherlands

e-mail: k.a.brookhuis@rug.nl

M. Dicke

Goudappel Coffeng BV,

Deventer, The Netherlands latter condition. After participants were provided with travel information, their driving performance with respect to the consequences of distraction differed between systems.

Conclusion The auditory information provision system allowed the best driving performance; the other two systems required the participants to look away from the road (too) long compromising safety, while reading an SMS took longer than scanning a PDA.

Keywords Nomadic systems · Travel information · Driving simulator $\cdot$ Driving performance

\section{Introduction}

In modern society, $24 \mathrm{~h}$ a day, 7 days a week, a host of information is available. Numerous messages of all kinds are distributed through telephones, computers, televisions, radios, for instance about travel opportunities. Transport companies and related business are starting to realize the feasibility and the financial benefits of providing travelers with information to make adequate choices while traveling. With the right resources and an adequate synchrony, it is technically possible to create a dynamic travel information provision system for travellers [7].

There are many situations in which it would be beneficial to know what to expect while traveling. Whether there is a fuel station, a traffic jam or a delayed train; all of these situations require reliable information and flexible means to reroute ourselves to solve the problem at hand. Commuters, professional travellers and even recreational travellers predominantly using a motor vehicle to travel, (would) greatly profit from extended travel information [9]; therefore the interest of this study was specifically how to provide car drivers with travel-information. In order to 
closely resemble an actual driving task while avoiding hazardous situations and controlling the environment, the study was conducted in a driving simulator.

Travel information can be presented to drivers in a variety of ways, through a number of available systems, depending on type of information, situation and recipient. When travelling in your own country, for instance, the information semblance may appear familiar irrespective of presentation mode, but travelling in a foreign country, local information may lead to misunderstanding and errors, and presentation mode might matter. One topic is what is the best feasible travel information system (off the shelf) when considering usability, safety, perception, comprehension and memory, and task demands? Another topic would be whether and how not only the obvious user of such systems (broadly 30 years old, used to electronic gadgets) appreciates travel information but also does a relative foreigner appreciate and understand it, i.e. is language proficiency a barrier in this respect.

To this end, travel information was presented to two different groups of participants with respect to language proficiency (natives and non-natives) in three different ways, through popular nomadic systems, a PDA, a mobile phone SMS and a mobile phone plugged in a car audio system, in two kinds of traffic conditions (calm and dense). The basic question before implementation and marketing should be how travel information could be presented optimally from a human factors point of view; however, automotive-electronic industry has introduced a variety of systems without any usability testing conditions or other involvement by the (Dutch) authorities. With a view to future integration of electronic systems in motor vehicles, potential problems with respect to human factors issues may be solved beforehand.

\section{Human factors principles for travel information}

When designing a system that aims to provide people with information while they are driving, it is essential to find a good match between the human information processing capabilities and information provision in the task environment. Mismatches between human being and task environment may cause misperceptions that might very well lead to accidents involving injury or death $[5,15]$.

Information processing, i.e. mental activities such as recognising, rehearsing, planning, understanding, decisionmaking and problem solving occur in working memory. Working memory is the temporary, effort demanding store where travel-information is kept active while or until we use it [16]. Travel-information can be provided by both visual and auditory means; it is important to know the limitations and possibilities of the information processing systems that have to do with the modalities of vision and audition. The limited processing capacity of working memory may cause a disruption in performance when two tasks are executed concurrently [1]. However, there might be little interference when two concurrent tasks entail information from different modalities [16]. Examples of this type of time-sharing are driving a car while trying to listen to the news on the radio at the same time or perceiving and understanding travel information.

The level of task automation has an influence on resource demands as well. In the present study, travel-information needs to be attended to during driving, the latter being a highly automated task for experienced drivers. The visual channel is most important for the driver and the concurrent execution of a visual task, like in-vehicle looking at a cell phone, competes for visual attention with the primary driving task [2]. But auditory (or motor or cognitive) tasks potentially create some conflict with monitoring and processing visual information as well. Because of this competition for limited resources, driving performance may decline. Alternatively, compensation may be sought by the driver, for instance by lowering speed. Therefore, several ways of providing information were investigated in order to find out if, and how, driving behaviour, performance and safety are affected. Additionally, the study aims to find out whether there are differences in information processing or in preferences between native Dutch speakers and non-native Dutch speakers.

\section{Method}

\subsection{Participants}

For the present study a total of twenty-four paid volunteers were recruited. In order to compare effects of language proficiency, native and non-native speakers of the Dutch language, two separate groups were selected. The first group of participants consisted of twelve people of nonDutch nationality ( 8 male and 4 female) with an average age of approximately 33 years. Participants in this group had been living in the Netherlands for 4.5 years on average. They were recruited at a School in Groningen with an adult education program for foreigners. The participants were taking Dutch lessons (2nd grade Dutch for Foreigners), third year students who graduated from level 2. The level of proficiency in the Dutch language of these participants was such that they were well able to understand all instructions, as well as provide reasonably adequate answers in Dutch. Their educational level was various, and country dependent. The group, referred to as 'non-natives' from now on, was selected on driving proficiency as well.

The second group of participants consisted of twelve Dutch volunteers ( 8 male and 4 female) with an average age of 26.3 years. All native participants were in possession of a Dutch driver's license and had more than 1 year of 
driving experience. They were recruited at the University of Groningen, students and young employees, all used to handle electronic systems.

\subsection{Apparatus}

To understand how different sources of information affect driver behaviour, the 'Driving Simulator' of the former Traffic Research Centre was used. The simulator was located at the academic hospital of Groningen, the "University Medical Centre Groningen", in the laboratory of the Neuro-Psychology department.

The simulator consists of a driver seat with steering wheel, three pedals, clutch and seatbelt, (partly) surrounded by three large screens, each two meters wide with a diameter of $4.5 \mathrm{~m}$. The driver seat is situated in front of the three screens, directly facing the middle screen; the two other screens are attached on the left and right of the middle screen in two 60-degree angles. Three beamers project the simulated world onto the screen, displaying the car's dashboard and rear-view mirrors, as well as the virtual reality outside the simulator. The position of the driver seat in front of the three large screens gives participants a 210 degree horizontal, and 60-degree vertical view on the virtual world projected before them.

Both the driver seat and beamers are connected to a central computer system, consisting of five interconnected personal computers (PCs); one central control PC with a Graphical User Interface, one PC that controls the traffic flow in the virtual world, and three PCs that render the virtual world on the left, middle and right screen. The PCs are interconnected through a Local Area Network (LAN). The software (StSoftware ${ }^{\circledR}$ ) used in the driving simulator contains several modules, one of which is a module for designing the layout of the simulator road environment. Another module is a script design tool to create conditions on the virtual route. The traffic module is a real-time simulation program, the render module renders the virtual world, and together they are the actual runtime modules.

A special feature of this particular simulator and its software is the use of the so-called 'autonomous agents' technique; all of the traffic in the virtual world exhibits autonomous, interactive behaviour. This means that the virtual vehicles in the simulation are not only capable of responding to each others' behaviour, they are also able to anticipate and react to the behaviour shown by the simulator-car. The specifics of the desired behaviour of the surrounding traffic can be set by programming through the scenario script design tool.

A customized virtual-reality driving environment was created, modelled after a standard motorway, to fit the explicit requirements of this study. This motorway was several kilometres long and had eight exits. Road signs indicating the appropriate destination marked each exit. Four of these exits led to make-believe cities with names such as 'Westdorp' or 'Noorddorp'. By taking such an exit, the participants were able to bypass potential congestion problems on the highway and reach the final destination, by secondary roads or 'Park and Ride' $(\mathrm{P}+\mathrm{R})$ alternative; the alternative means that participants were offered the option to park their car and continue their journey by means of public transportation.

\subsection{Procedure and design}

The present study was composed of one between-subjects factor, i.e. language proficiency, and two within-subjects factors, i.e. information provision system and traffic density. Providing information compares the three different ways participants in this study were receiving information, i.e. by mobile phone SMS (using short text messages on a Nokia 3310 mobile phone), by PDA/palmtop (HewlettPackard IPAQ Pocket PC), and by auditory messages (using recorded voices over the car audio system). The density of the surrounding traffic had two levels, busy $(1,000$ vehicles/h) and calm (600 vehicles/h). Each participant was required to complete six trials for this reason.

To avoid learning and sequence effects, six different scenarios were presented in the six trials to all participants in which different problems asked for different solutions, the order in which scenarios were presented to the individual participants was according to a Latin square, cross-over distributed across the three systems and two densities. The scenarios involved three types of problems (traffic jam, road constructions, accident) with two general types of solutions (take a secondary road or go to the $\mathrm{P}+\mathrm{R}$ and take the bus). Although the delays caused by problems in different scenarios varied (20-30 $\mathrm{min})$, the alternative offered by the information system always shortened the delay by $15 \mathrm{~min}$.

Prior to the onset of each experiment, every participant received a brief introduction to the study, an informed consent, and all the questionnaires used for practice. After participants read the introduction and completed an initial questionnaire (asking them about their age, nationality, gender, etc.) they received instructions about how to read an SMS on the mobile phone, how to read the information on the PDA, and they were introduced to the voice messages giving them the route information the auditory way. To familiarise with the virtual reality of the simulator they were thereupon first asked to complete a practice session in the simulator for about $10 \mathrm{~min}$. Allowing the participants to get acquainted with driving the simulator gave the researcher a chance to check for possible simulator sickness. The participants were instructed to drive as they would normally do, following the motorway until the 
message occurred after which they could decide to continue or take the exit.

Including the ten-minute practice trial, the total amount of time a participant spent inside the simulator was about $40 \mathrm{~min}$. After each of the six trials, the participant was asked to complete a couple of questionnaires that inquired about usability and comfort of the way the information was presented. Participants were also interviewed about their own driving-performance and their perceived workload during the previous trial.

\subsection{Information provision}

In each of the six trials, $1,500 \mathrm{~m}$ before the exit that led to the alternative route, the computer emitted a sound over the loudspeakers that alerted the participant to attend to the incoming information. In both SMS and PDA conditions, participants were then required to press a button to display the information (for each display in turn). In the auditory condition the information followed automatically after the tone, similar to the well-known RDS-TMC.

The mobile phone for short text messages (SMS) and the PDA were located in the simulator near the participant by means of a 'car kit', allowing hands-free operation, the legal requirement in the Netherlands. Previous research has shown that the best way to present route information on a mobile phone is to divide the information into small, meaningful 'chunks', displaying a specific part of the entire message [8]. An example of what this looked like is shown in Fig. 1 (translated into English). On the PDA information was presented in small 'chunks' as well, consisting of three general themes central to each message (problem, consequence and option). Pictograms (such as a $\mathrm{P}+\mathrm{R}$ sign and a bus) were incorporated in the messages on the PDA, making the message shorter, clearer, and more universal, see Fig. 1 for an example (in Dutch).

In three auditory messages, the information was divided into three small and meaningful units, similar to the way this was done for the SMS and the PDA screens. The
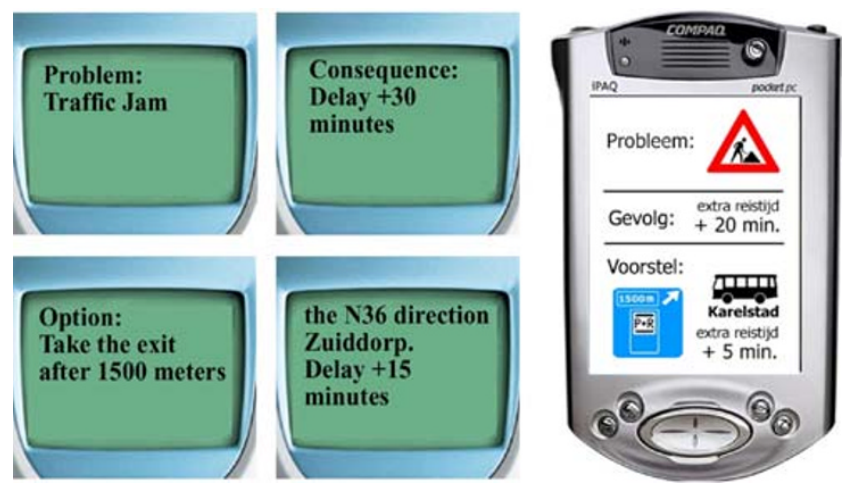

Fig. 1 Example of a scenario displayed on a mobile phone and on PDA (in Dutch) spoken texts were pre-recorded, and uploaded into the 'digital script' of the simulator. To enhance the messages and distinguish between the three chunks of information, two different voices were recorded; a male voice and a female voice. The male voice stated the title of each chunk of information ('problem', 'consequence', 'option') and the female voice gave the relevant information.

\subsection{Dependent variables}

Dependent variables were collected from three different sources. Participants were requested to complete a number of questionnaires, they were filmed during the experiment with the aim to record eye movements, and additionally a number of driving performance measures were collected and saved on the simulator computer.

Self-report measures were used to gain insight into the (experienced) workload, the (self-evaluation of) driving performance, and the (perceived) usability and comfort of the three systems by which travel-information was provided. To check whether the participants understood the route information and remembered the message, they were asked what they remembered from its content.

\subsubsection{Self-reported mental workload}

To measure cognitive workload under the different conditions of this study, all participants were required to fill out the rating scale of mental effort (RSME) after each trial, a Dutch self report scale at an interval level [17], from $0=$ no effort, to $100=$ very much effort.

\subsubsection{Self-reported driving performance}

Participants were asked to rate their self-perceived driving performance after each trial. This measure gives an indication on how different manipulations affect the way participants believed they were driving [3], from $-5=$ very poor to $+5=$ very well.

\subsubsection{Acceptance}

Acceptance of the systems was measured with a subjective nine-item scale to measure specific acceptance in two dimensions; a usability dimension and a comfort dimension ([14]), while after the participants completed the experiment, a second, general usability scale measure was acquired.

\subsubsection{Memory}

To test how much of the information provided was remembered, participants were asked after each trial what they remembered from the messages that contained six 
items each, hence, the minimum score on this variable was 0 and the maximum was 6 . The score derived is considered an indication of how well the drivers actually perceived, processed and understood the travel information while they engaged in driving.

\subsubsection{Objective measures of safety}

Perhaps the most important factor that needs to be considered when using a system designed to provide information while driving is safety. Several studies have demonstrated that when using support systems at the manoeuvre level, such as collision warning, driving performance improves, while support systems at the strategic level, such as information provision about detours, may compromise safety (see [4]). In this study, safety related driving performance was assessed by using specific objective measures. Two captures of driving behaviour were used as an indicator for driving performance and safety around the critical period (i.e. shortly before and after the message providing information); speed and time-headway to cars-in-front. The two simulator variables were measured for a total duration of $45 \mathrm{~s}$ before and $45 \mathrm{~s}$ after introduction of the auditory signal warning the participants of incoming travel-information.

Driving a car at $120 \mathrm{~km} / \mathrm{h}, 33.3 \mathrm{~m}$ of highway is covered during each and every second that the driver's gaze drifts off the road. To assess the potential danger of mobile phone or PDA usage in this sense (cf. [12]), both the number of times and the total amount of time each participant looked away from the road (while reading the information on the screen) was measured through video-analysis.

\section{Results}

\subsection{Self-reports}

\subsubsection{Workload scale}

The experienced workload is depicted in Fig. 2. The difference in experienced workload between the two groups

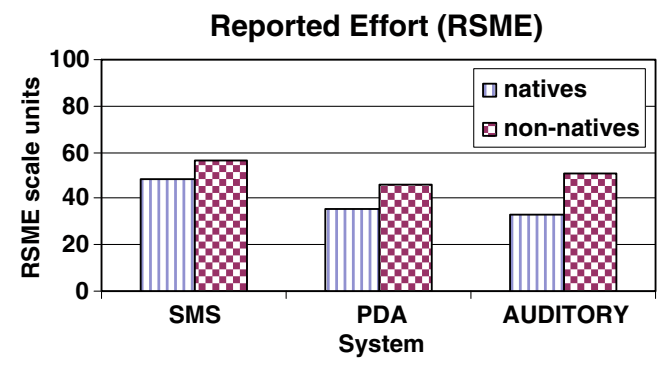

Fig. 2 Comparing the two groups on self-reported effort $(0=$ no effort, $100=$ extreme effort) of participants shows a small but non-significant effect. Natives appear to experience less workload while driving the simulator than do non-natives. A post-hoc analysis indicates that only the native participants show a significant effect for the way information is provided $(\mathrm{F}(1,11)=4.2$, $P<0.028$ ). Providing information by SMS differs significantly from the other two systems in the sense that these participants experienced more workload in the SMS condition compared to the other two systems.

\subsubsection{Driving performance self-report}

The system used for providing information had a small non-significant effect on how people in this study evaluated their own driving performance $(\mathrm{F}(1,22)=2.8, P<0.07)$. Participants tended to evaluate their own driving as worst when information was provided to them by SMS, and to believe their performance was best when information was provided by sound.

\subsubsection{Usability and comfort factors in the acceptance scale}

Acceptance of each system was differentiated in two dimensions, usability and comfort. Prior to experience, i.e. before driving in the simulator, and after each trial in the simulator participants were given the acceptance scale through which they were asked to indicate how useful and comfortable they thought an information-provision system (as described or experienced) would be to them.

The small difference that was found indicates that the native participants evaluated the information-providing systems as less useful than did the non-natives (Fig. 3). Worth mentioning is that the usability scale measured prior to the research trials (the anticipated usability) already showed a similar difference between the two groups. For the dependent variable 'usability', a small non-significant interaction $(\mathrm{F}(1,22)=2.8, P<0.07)$ was found between the independent variables language proficiency, system and traffic density.

When looking at the graph in Fig. 4, it is clear that the two groups of participants differ in the degree of comfort they experienced while information was provided to them.

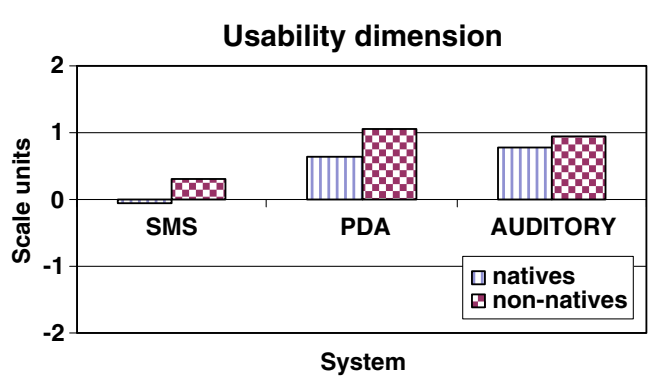

Fig. 3 Comparing groups and systems on usability (scale from -2 to 2) 


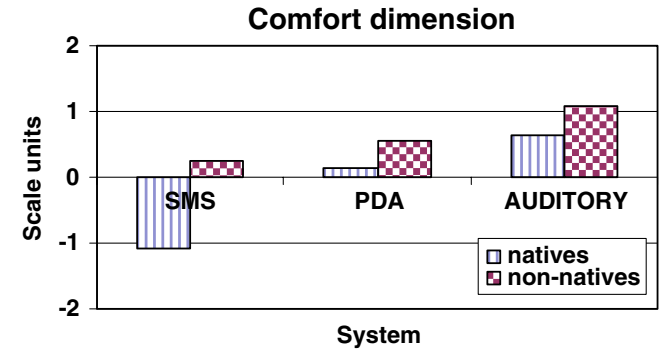

Fig. 4 Comparing groups and systems on comfort (scale from -2 to 2)

Non-natives experienced higher levels of comfort than natives $(\mathrm{F}(1,22)=6.1, P<0.022)$, with a small nonsignificant interaction $(\mathrm{F}(1,22)=2.7, P<0.08)$ with the system used. The two groups seem to differ more in their opinion of the degree in which a system is comfortable in the case where information is received by SMS. The systems used to provide information were evaluated significantly different with respect to how comfortable they were $(\mathrm{F}(2,21)=14.9, P<0.001)$.

\subsubsection{Memory}

Traffic density seems to have only a small non-significant effect on the number of items remembered $(\mathrm{F}(1,22)=3.5, P<$ 0.06 ), contrary to expectations. However, the difference in remembered items between the two groups of participants shows a significant effect $(\mathrm{F}(1,22)=8.7, P<0.007)$. Natives remembered more items from the information provided to them than did non-natives, while no difference was found between the three systems (Fig. 5).

\subsection{Objective measures, performance and safety}

\subsubsection{Speed}

The two participant groups differed slightly but not significantly in the speed they kept. After the warning signal was presented and participants were required to attend to the provided travel-information, they reduced their speed considerably, by about $7 \mathrm{~km} / \mathrm{h}(\mathrm{F}(1,22)=39.6, P<$ 0.001 ), differently for the various conditions, see Table 1 .

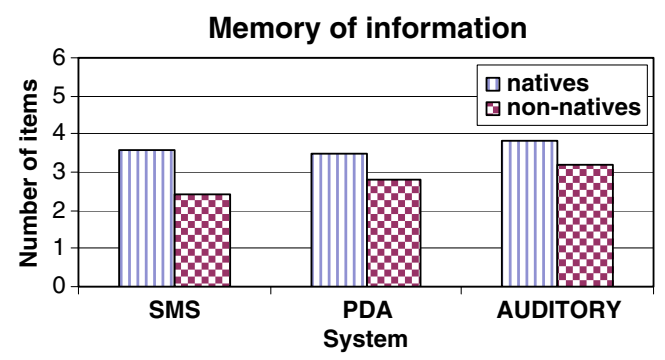

Fig. 5 Average number of items remembered for each group (from 0 to 6 )
A rather small, non-significant effect was found for the system by which information was provided, whereas a large effect was found for traffic density on mean driving speed $(\mathrm{F}(1,22)=12.2, p<0.002)$. Participants tended to drive significantly faster when traffic density was low, compared to the situation in which traffic density was high, thus showing a larger decrease directly after the information provision.

\subsubsection{Time headway}

Mean time headway varied from 1.49 up to $4.77 \mathrm{~s}$ throughout the different conditions. It turned out that time headway only slightly increased after the signal but not significantly. Thus the average time distance participants kept did not change after the signal indicative of incoming information. However, the minimum time headway, a relevant indicator of safe driving, did change while processing incoming information $(\mathrm{F} 1,22)=5.1, P<0.034$, while a significant interaction with traffic density $(\mathrm{F}(1,22)=$ 5.8, $P<0.024)$ indicates that only in the busy traffic condition minimum time headway increased, from $.70 \mathrm{~s}$ to $.93 \mathrm{~s}$. A higher percentage of time headway under $5 \mathrm{~s}$ also indicates less safe driving behaviour. With respect to the three different systems, no significant effect was found but traffic density did have a significant effect $(F(2,21)=7.3$, $P<0.013)$ too. In busy traffic, participants drive relatively close to the car in front of them as compared to under calm traffic conditions, but similar to the manifest speed adaptation, participants adapted their safety margins to the car in front.

\subsubsection{Duration of eye fixations}

To investigate how long it takes for a participant to read and understand the message provided to them, the total amount of time they shifted their gaze off the road to the information providing device was analysed. Figure 6 indicates that when participants received the information by SMS, they looked away from the road and at the system for a longer total amount of time than when they received the information on the PDA $(\mathrm{F}(1,22)=15.1, P<0.003)$. Density of the surrounding traffic did not have an effect at all, while the two groups differed slightly in the total amount of time their gaze was directed away from the road $(\mathrm{F}(1,22)=3.9, \quad P<0.06)$. From Fig. 6 it can be concluded that, as might be expected, non-natives take more time to inspect the message presented to them, but with considerable variation $(\mathrm{SD}=7.9$ and $\mathrm{SD}=6.8$ respectively).

The average duration of each eye fixation differed considerably as well when the information is provided by SMS, $1.2 \mathrm{~s}$, as compared with the PDA, $1.0 \mathrm{~s}(\mathrm{~F}(1,22)=$ $15.6 P<0.001)$. 
Table 1 Mean speed $(\mathrm{km} / \mathrm{h})$ before and after the signal indicating incoming information

\begin{tabular}{|c|c|c|c|c|c|c|}
\hline & \multicolumn{2}{|l|}{ SMS } & \multicolumn{2}{|l|}{ PDA } & \multicolumn{2}{|c|}{ AUDITORY } \\
\hline & pre & post & pre & post & pre & post \\
\hline Natives (Busy) & 95.8 & 96.9 & 93.6 & 90.1 & 96.9 & 93.3 \\
\hline Natives (Calm) & 112.7 & 111.9 & 108.2 & 98.1 & 107.2 & 92.7 \\
\hline Non-Natives (Busy) & 88.5 & 73.2 & 90.7 & 86.3 & 92.9 & 88.8 \\
\hline Non-Natives (Calm) & 102.8 & 89.2 & 94.9 & 86.1 & 97.2 & 92.7 \\
\hline Total & 99.9 & 92.8 & 96.8 & 90.1 & 98.6 & 91.9 \\
\hline
\end{tabular}

\section{Discussion}

The total of 24 participants is sufficient for a power of .95 at alpha level .05; they were representative of relatively young adult drivers in the Netherlands that are comfortable with mobile phones and other electronic gadgets. However, the division in two groups necessitates careful and reserved statements about differences in language proficiency. Only sufficient differences between the two groups are to be considered.

When comparing the systems used for providing information, SMS is consistently evaluated as the least usable and comfortable. The other two systems generally do not differ much from one another, but when they do, auditory information provision is evaluated as being most comfortable. Non-native speakers consistently view the information-providing systems as more useful and comfortable than natives do. This might be attributed to a genuine difference between the two groups, because the non-native participants would benefit more from such a system. Nonnatives also tend to evaluate the SMS better than natives do, which might be caused by the fact that with an SMS it is possible to read the message more than once, which is beneficial when you are not fluent in the language at hand. As was expected, the degree of traffic density didn't have an effect on usability.

It was hypothesized that visual systems would compete more strongly for attention with the driving task than the auditory system. But only SMS was found to cause higher experienced workload. From this it can be concluded that

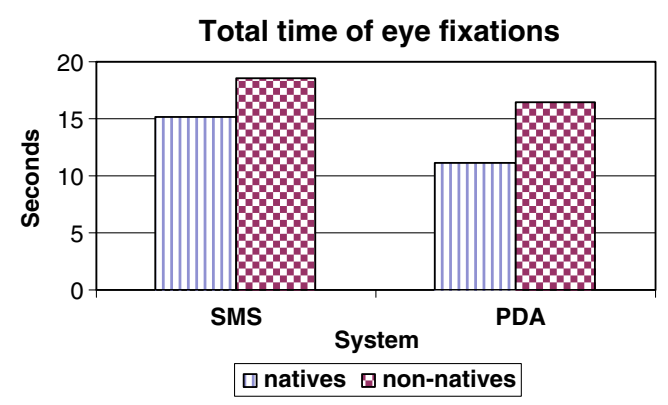

Fig. 6 Duration of time the two groups attended to the information providing system pictograms (PDA) and auditory information work better than SMS does. Memory performance wasn't affected at all by the type of system used. Natives did remember more of the Dutch travel-information as was expected in SMS and auditory conditions, but they also remembered more when symbols were used (PDA). A confounding factor may be the level of education, which was generally lower for nonnative participants.

When participants were asked about their own driving performance, they believed they drove best when an auditory system presented the information, slightly worse when a PDA was used, and worst when an SMS was used. Although this is consistent with the results found for usability, the objective driving performance measures did not show that the type of system used had an effect on driving performance. Driving performance, and thus safety, doesn't seem to be affected much by the type of system used. However, when comparing SMS and PDA with respect to the amount of time participants looked away from the road, there is a difference between the two. As expected, drivers took longer to look at an SMS than they did with a PDA, implying that a PDA is safer than an SMS, however, both systems might still compromise safety. Previous research has found that drivers feel safe when glances are shorter than $0.8 \mathrm{~s}$ [10]. In this study, looking at the SMS takes about $1.2 \mathrm{~s}$, and looking at the PDA occupies participants close to a full second. Moreover, specifically SMS violated the 15 second-rule [10], i.e. the total time needed for acquiring information from a device should not exceed $15 \mathrm{~s}$, be it that the rule holds for nonmoving vehicles. A possible solution to this problem might be to focus on creating a more user-friendly design for the displays, or by making use of auditory displays. However, even verbal tasks still lead to some degree of interference [11] and they do not eliminate distraction altogether [13].

\section{Conclusion}

The present study shows that reading an SMS on a mobile phone while driving, even if it is placed in a car-kit, is not safe. Despite this finding, the mobile phone is used predominantly by the current traveler population. Never- 
theless, new technological advances are a big step forward compared to the old paper roadmap. In this study, PDA and auditory provision of information were found to be relatively safe ways to provide drivers with the information they need. The other side of the coin is that many advances in technology may also cause substantial distraction to the driver. Cohen and Graham [6] have estimated that eliminating the use of mobile phones would save 2,600 lives and prevent 330,000 injuries annually in the U.S. alone. Information is power, but at what price...

Acknowledgement This study was carried out in the framework of the Dutch Transumo Program (www.transumo.nl)

\section{References}

1. Baddeley AD, Hitch GJ (1974) Working Memory. In: Groome D (ed) An introduction to cognitive psychology, vol. 5. Psychology, East Sussex, UK

2. Brookhuis KA, De Vries G, De Waard D (1991) The effects of mobile telephoning on driving performance. Accid Anal Prev 23:309-316

3. Brookhuis KA, De Vries G, Prins van Wijngaarden P, Veenstra G, Hommes M, Louwerens JW, O'Hanlon JF (1985) The effects of increasing doses of Meptazinol (100, 200 and $400 \mathrm{mg}$ ) and Glafenine (200 mg) on driving performance. Research report VK85-16, Haren: Traffic Research Centre, University of Groningen

4. Carsten O, Brookhuis KA (2005) The relationship between distraction and driving performance: towards a test regime for in-vehicle information systems. Transp Res Part F 8:191-196

5. Casey S (1993) Set phasers on stun and other true tales of design, technology and human error. Aegean, Santa Barbara, C.A., USA
6. Cohen JT, Graham JD (2003) A revised economic analysis of restrictions on the use of cell phones while driving. Risk Anal 23:114

7. De Jong C, Brookhuis KA (2005) De haalbaarheid van betrouwbare reisinformatie. (The feasibility of reliable travel information). Report to ProRail (Dutch Railways), Groningen: University of Groningen

8. Dicke M, De Groot JIM (2005) Passende oplossing voor reisinformatie op mobiele telefoons. In: Clement R (ed) Fitting solution for travel information on mobile phones. Colloquium Vervoersplanologisch Speurwerk, Rotterdam, pp 1687-1706

9. Dicke, M. (2008). Safe and innovative travel information. TRAIL Research School PhD Thesis Series, Delft: Delft University of Technology

10. Green PA (1999) Visual and task demands of driver information systems (UMTRI 98-16). University of Michigan Transportation Research Institute, Ann Arbor

11. Lee JD, Caven B, Haake S, Brown TL (2001) Speech-based interaction with in-vehicle computers: the effect of speech-based email on drivers' attention to the roadway. Hum Factors 43:631-640

12. Mourant RR, Rockwell TH (1972) Strategies of visual search by novice and experienced drivers. Hum Factors 14:325-335

13. Strayer DL, Drews FA, Johnston WA (2003) Cell phone-induced failures of visual attention during simulated driving. J Exp Psychol-Appl 9:23-32

14. Van der Laan JD, Heino A, De Waard D (1997) A simple procedure for the assessment of acceptance of advanced transport telematics. Transportation Research Part C 5:1-10

15. Wickens CD, Hollands J (2000) Engineering psychology and human performance, 3rd edn. Prentice Hall, Upper Saddle River, N.J., USA

16. Wickens CD, Lee JD, Liu Y, Becker SEG (2004) An introduction to human factors engineering, 2nd edn. Prentice Hall, Upper Saddle River, N.J., USA

17. Zijlstra FRH (1993) Efficiency in work behavior. A design approach for modern tools. PhD Thesis, Delft University of Technology. Delft University Press, Delft, The Netherlands 